Article

\title{
Phaseless Radar Coincidence Imaging with a MIMO SAR Platform
}

\author{
Aaron V. Diebold *(D, Mohammadreza F. Imani ${ }^{\circledR}$ and David R. Smith \\ Department of Electrical and Computer Engineering, Duke University, Durham, NC 27708, USA; \\ mohamad.imani@gmail.com (M.F.I.); drsmith@ee.duke.edu (D.R.S.) \\ * Correspondence: aaron.diebold@duke.edu
}

Received: 8 January 2019; Accepted: 27 February 2019; Published: 5 March 2019

\begin{abstract}
The correlation-based synthetic aperture radar imaging technique, termed radar coincidence imaging, is extended to a fully multistatic multiple-input multiple-output (MIMO) synthetic aperture radar (SAR) configuration. Within this framework, we explore two distinct processing schemes: incoherent processing of intensity data, obtained using asynchronous receivers and inspired by optical ghost imaging works, and coherent processing with synchronized array elements. Improvement in resolution and image quality is demonstrated in both cases using numerical simulations that model an airborne MIMO SAR system at microwave frequencies. Finally, we explore methods for reducing measurement times and computational loads through compressive and gradient image reconstruction using phaseless data.
\end{abstract}

Keywords: ghost; RCI; correlation; radar; coincidence; imaging; MIMO; SAR

\section{Introduction}

Radar coincidence imaging ( $R C I)$ is a recently proposed microwave imaging approach that applies principles of ghost imaging in a radar array or synthetic aperture radar (SAR) framework [1]. Whereas conventional SAR systems employ a single transceiver to interrogate a target as the radar platform moves along a synthetic aperture path (monostatic acquisition), a hallmark of RCI is the simultaneous use of a collection of transmitters and/or receivers for rapid multistatic signal acquisition. To date, multiple-input single-output (MISO) [1-4] or single-input multiple-output (SIMO) [5] RCI systems have been put forth as candidates for achieving incoherent measurement patterns required in a ghost imaging scheme through a superposition of approximately time- and space-independent waveforms. In these cases, approximately uncorrelated measurement patterns can be guaranteed through stochastic modulation of the transmitted signals. This offers the added benefit of introducing natural anti-jamming qualities to the radar system [6].

The ability to implement RCI in MISO or SIMO configurations indicates that it can be handled more generally under a fully multiple-input multiple-output (MIMO) formulation. MIMO radar involves leveraging the large number of degrees of freedom offered by transmitting and receiving arrays for improved system performance in terms of resolution or detection capabilities $[7,8]$. Instead of sequentially switching through each channel in the MIMO system, significant effort has gone into investigating orthogonal coding schemes in order to access these additional degrees of freedom for post processing [8-13]. This enables rapid signal acquisition through simultaneous illumination and reception among all of the array elements, with the potential for high-speed and high-resolution, wide-swath radar. In proposed systems, exploiting the additional degrees of freedom for improved image resolution requires coherent processing [8], which demands sophisticated synchronization capabilities between the transmitting and receiving array elements [14-17]. In answer to the above requirements, RCI can handle the signal extraction problem naturally through a 
correlation-based image reconstruction method that is well-established in multiplexing computational imaging systems [18]. The further degrees of freedom of the MIMO system in this case enter into the problem implicitly through the spatial incoherence of the combined illuminating fields, which become approximately orthogonal in the limit of a dense transmitting array. In addition, by noting the relationship of RCI to the optical ghost imaging method, we will see that incoherent processing of RCI data can significantly alleviate synchronization requirements for high-resolution MIMO imaging.

In optical ghost imaging experiments, an image is recovered through a correlation between the spatially varying transmitted intensity patterns and a set of backscatter intensity measurements collected by a single detector [19]. Computational ghost imaging [20,21] employs known, computable patterns, and the correlation is performed with respect to a set of distinct patterns. Such an architecture achieves lenseless, single-pixel imaging directly from intensity measurements, which can be highly advantageous given the restrictions of optical detection hardware. Nevertheless, the relative ease of measuring phase at microwave frequencies has encouraged reconstruction through full-field (phase and amplitude) correlations, or other inversion techniques, in most RCI demonstrations. In this context, RCI can be viewed as a multistatic implementation of time-domain correlation with pulse diversity [6]. While it is true that full-field correlations can achieve improved image convergence rates over their phaseless counterparts [22], several unique advantages have been documented for the phaseless optical ghost imaging approach [23-25] that have not been adequately explored in the microwave radar setting. These include a robustness to turbulence on the receive arm and the removal of synchronization requirements between source and receiver. In addition, RCI proposals have primarily utilized ground-based radar configurations. With the advent of metasurface antennas, many of the daunting hardware bottlenecks facing both ground-based and airborne MIMO SAR systems can be circumvented [26,27]. Dynamic metasurface apertures [28-32] in particular stand as a versatile, low-cost and lightweight technology that can facilitate the necessarily large-scale framework supporting MIMO SAR techniques. We thus propose RCI on a MIMO SAR platform, investigate the differences between full-field and phaseless modalities, and identify advantages of each scheme. In particular, we show that full-field reconstruction achieves superior imaging performance, while phaseless reconstruction can alleviate hardware synchronization requirements as well as computational demands and phase error artifacts in reconstructions. Motivated by the advantages offered by phaseless imaging, we also investigate compressive techniques. Furthermore, considering the inherent motion of an airborne SAR system, we propose an approach which leverages array motion to directly achieve edge detection of targets. Its advantages are examined in the context of phaseless and compressive reconstruction. While the economic advantages of the aforementioned technologies help motivate this analysis, we remain agnostic to the particular hardware approach and focus on new acquisition and processing possibilities enabled by MIMO SAR.

In what follows, we detail the MIMO SAR imaging configuration as well as the RCI reconstruction formulation. We investigate the effects of array geometry on the imaging point spread function and the resulting imaging performance. Phaseless and full-field correlation reconstructions are compared, as well as results of applying more advanced reconstruction techniques. Finally, we outline various system differences between the two cases, such as synchronization requirements and effects of phase error.

\section{Results}

\subsection{Imaging Configuration}

The imaging geometry employed in this work is shown in Figure 1. For the sake of presentation, we consider in this paper an airborne MIMO SAR scenario in which a collection of $N=40$ airborne transmitters (investigated in Section 2.2.1), e.g., a constellation of transmitting UAVs, fly at an altitude of $x=2 \mathrm{~km}$ in the $y z$ plane, parallel to the two-dimensional region of interest. We assume all transmitters at a given time are constrained within a 200 by $200 \mathrm{~m}^{2}$ area with positions given by $\vec{r}_{T n}(t)$ with 
$n=1, \ldots, N$, where we allow the transmitter positions to vary with time to reflect the fact that different pulses may originate from different positions along the constellation trajectory. Each transmitter traverses a total distance of $40 \mathrm{~m}$. We make a stop-and-shoot approximation throughout this work, neglecting effects of motion during a pulse and assuming stationary transmitters/receivers during a pulse. The number of receivers $K$ within the constellation will vary depending on the implementation, and their positions will similarly be allowed to vary in time.

(a)

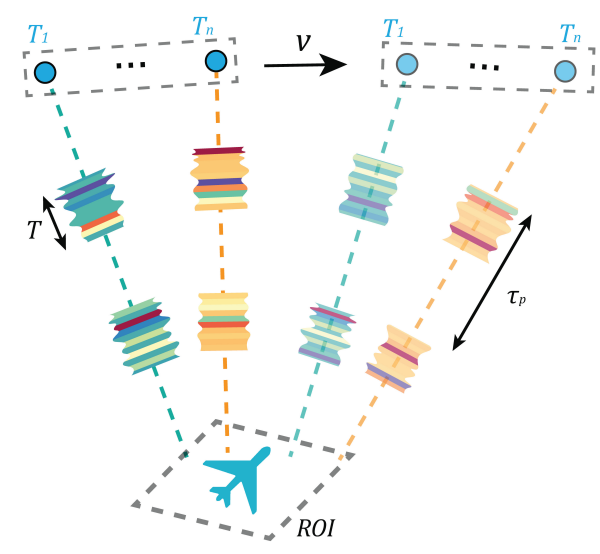

(b)

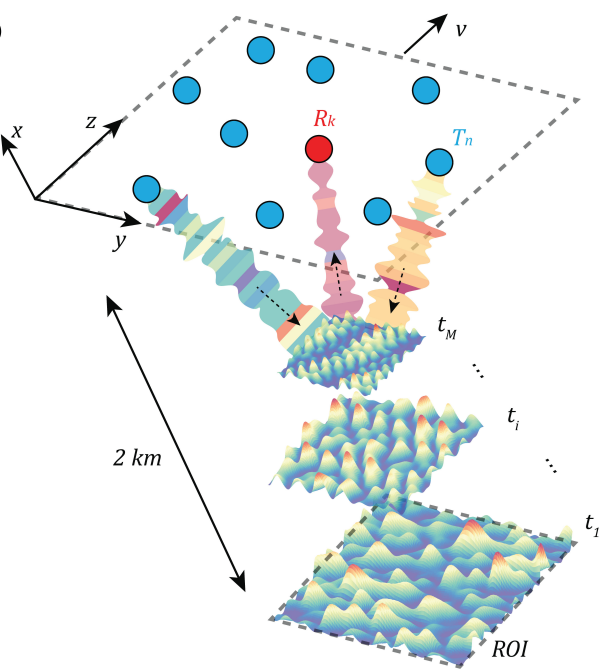

Figure 1. (a) Side view of layout for airborne radar coincidence imaging (RCI). (b) Diagonal view of layout for airborne RCI, including example intensity patterns at different times within a pulse.

Each transmitting source emits an amplitude-, phase- and frequency-modulated pulsed signal with a frequency range of 9.5-9.8 GHz sampled at $15 \mathrm{MHz}$. The pulse waveform emitted from the $n^{\text {th }}$ transmitter, $s_{n}(t)$, is modeled as [1]

$$
s_{n}(t)=\sum_{q=1}^{Q} A(t) \operatorname{rect}\left(\frac{t-q \tau_{p}}{T}\right) \exp [j(2 \pi f(t) t+\phi(t))]
$$

where the amplitude $A(t)$, frequency $f(t)$ and phase $\phi(t)$ are stochastically-varying with uniform distributions on the intervals $[0,1],[9.5 \mathrm{GHz}, 9.8 \mathrm{GHz}]$ and $[-\pi, \pi]$, respectively. These parameters fluctuate within a single pulse, as well as over the entire waveform/acquisition time (i.e., across all constellation positions). $T$ indicates the pulse duration, which is taken to be $1 \mu \mathrm{s}$, and $\tau_{p}$ is the pulse repetition interval. $q=1, \ldots, Q$ indexes a single pulse within the waveform, so that $Q \tau_{p}$ is the total acquisition time. Unless otherwise stated, we model the acquisition process with a single transmitted pulse per each equispaced constellation position, for a total of $Q=50$ pulses/positions. The transmitters are assumed to be phase-synchronized, and illuminate the scene simultaneously. Under this condition, the superposition of their pulses, $s(\vec{r}, t)$, at each position $\vec{r}$ of the imaging domain can be computed for every instance of time $t$ :

$$
s(\vec{r}, t)=\sum_{n=1}^{N} s_{n}\left(t-\frac{\left|\vec{r}-\vec{r}_{T n}(t)\right|}{c}\right)
$$

where $c$ is the speed of light.

The scene to be reconstructed is represented by a spatially-varying scattering density $\sigma(\vec{r})$ that occupies a $4 \mathrm{~m}$ by $4 \mathrm{~m}$ area. While such a constrained region of interest is not practically achievable, the results obtained for this scenario can be generalized through proper scaling and sampling. We restrict ourselves to such a limited area in this work due to computational limitations. 
We assume a non-fluctuating target model, so that $\sigma(\vec{r})$ is a deterministic, unknown quantity [33]. The scattered signal, under the first Born approximation, is $s(\vec{r}, t) \sigma(\vec{r})$. The signal $s_{R k}(t)$ measured by receiver $k, k=1, \ldots, K$, located at position $\vec{r}_{R k}(t)$, is given by a sum over this scattered signal modified by the appropriate propagation delay:

$$
\begin{aligned}
s_{R k}(t) & =\int \sigma(\vec{r}) s\left(\vec{r}, t-\frac{\left|\vec{r}_{R k}(t)-\vec{r}\right|}{c}\right) d V \\
& =\int \sigma(\vec{r}) \sum_{n=1}^{N} s_{n}\left(t-\frac{\left|\vec{r}-\vec{r}_{T n}(t)\right|}{c}-\frac{\left|\vec{r}_{R k}(t)-\vec{r}\right|}{c}\right) d V \\
& =\int \sigma(\vec{r}) s_{r e f, k}(\vec{r}, t) d V
\end{aligned}
$$

where $V$ indicates the region of interest to be imaged, and the reference signal for receiver $k, s_{r e f, k}$, is defined as

$$
s_{r e f, k}(\vec{r}, t)=\sum_{n=1}^{N} s_{n}\left(t-\frac{\left|\vec{r}-\vec{r}_{T n}(t)\right|}{c}-\frac{\left|\vec{r}_{R k}(t)-\vec{r}\right|}{c}\right) .
$$

When $N$ is sufficiently large, these reference fields are expected to be spatially incoherent [1] and to approximately obey speckle statistics [34], while temporal incoherence is guaranteed through the stochastic fluctuations of each transmitted signal. Spatial and temporal incoherence are characterized by the condition

$$
\kappa \int s_{r e f, k}^{*}(\vec{r}, t) s_{r e f, k}\left(\vec{r}^{\prime}, t+\tau\right) d t \approx N \delta\left(\vec{r}-\vec{r}^{\prime}\right) \delta(\tau) .
$$

where we are considering our reference fields to be stationary in time. $\kappa=\left(\int_{t} d t\right)^{-1}$ is a normalization factor characterizing the length of the acquisition interval. In the remainder of this work, we will generally ignore constant normalization terms unless required for clarity. The magnitudes of two example reference patterns are depicted in Figure 2a,b, illustrating the speckle nature of the fields for large $N$.
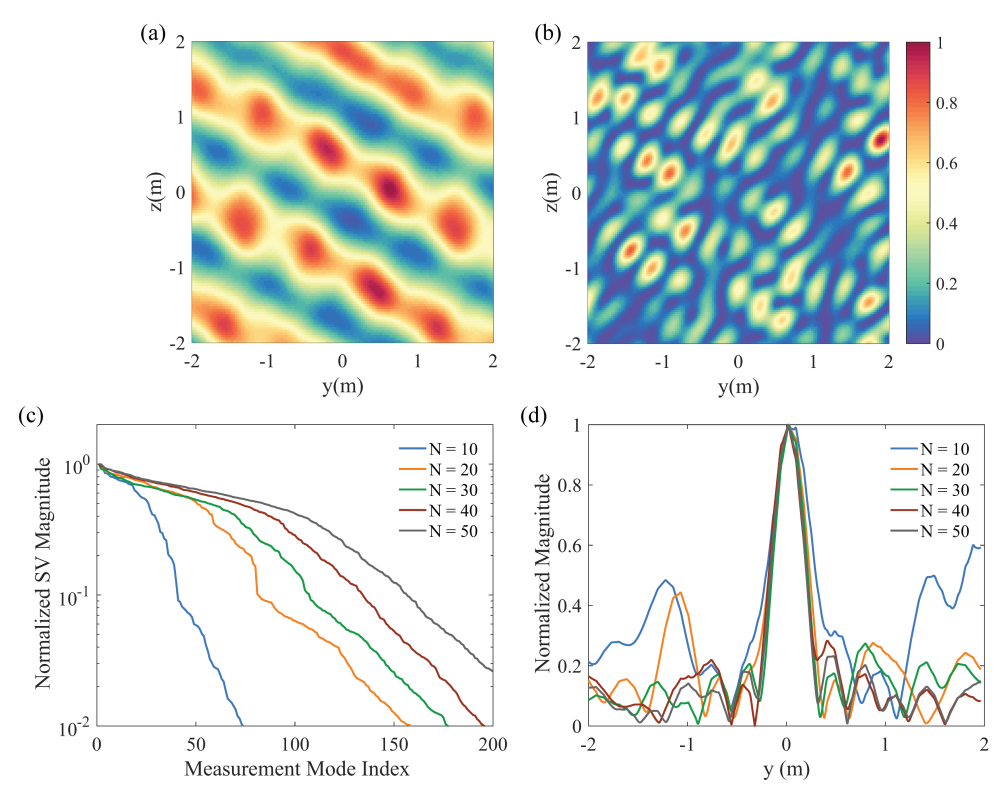

Figure 2. (a) Example reference pattern for $N=10$ transmitters. (b) Example reference pattern for $N=40$ transmitters. (c) singular value decomposition (SVD) of complex reference fields as $N$ is varied. (d) y-slice of center-position spatial autocorrelation as $N$ is varied. 
For computational purposes, we can equivalently define these reference fields in the frequency domain as

$$
S_{r e f, k}\left(\vec{r}, f, t_{s}\right)=\sum_{n=1}^{N} S_{n}\left(f, t_{s}\right) \frac{\exp \left[-\frac{j 2 \pi f}{c}\left\{\left|\vec{r}-\vec{r}_{T n}\left(t_{s}\right)\right|+\left|\vec{r}_{R k}\left(t_{s}\right)-\vec{r}\right|\right\}\right]}{\left|\vec{r}-\vec{r}_{T n}\left(t_{s}\right)\right|\left|\vec{r}_{R k}\left(t_{s}\right)-\vec{r}\right|}
$$

where $S_{n}\left(f, t_{s}\right)$ is the temporal Fourier transform of $s_{n}(t)$ with respect to the fast time (i.e., the signal temporal variation), and $t_{s}$ denotes the slow time that characterizes the constellation motion.

\subsection{Reconstruction}

\subsubsection{Single Receiver}

Several works to date have demonstrated RCI using a single-receiver $(K=1)$ configuration [1-4]. We similarly choose this case as our starting point, since many characteristics of the RCI approach can be intuitively understood and adequately described under such a configuration. We denote the single receiver position $\vec{r}_{R}(t)$, its measured signal $s_{R}(t)$ and its reference signal $s_{r e f}(\vec{r}, t)$. In RCI using complex measurements, an image $G_{c}(\vec{r})$ is obtained through a complex cross-correlation between the measured signal and the reference signals. In the time domain, this correlation is

$$
G_{c}(\vec{r})=\int_{t} s_{r e f}^{*}(\vec{r}, t) s_{R}(t) d t
$$

In Equation (7), the correlation is performed over the total acquisition time $Q \tau_{p}$, which includes all positions traversed by the array. Under the large $N$ assumption, $s_{r e f}(\vec{r}, t)$ is spatially incoherent [1]. For stochastic signals, the large $N$ assumption further guarantees circular complex Gaussian statistics, which ensures the spatial incoherence of the reference field intensities through the complex Gaussian moment theorem [22] paired with Equation (5):

$$
\begin{aligned}
\int_{t}\left|s_{r e f}(\vec{r}, t)\right|^{2}\left|s_{r e f}\left(\vec{r}^{\prime}, t\right)\right|^{2} d t & =\kappa\left|\int_{t} s_{r e f}^{*}(\vec{r}, t) s_{r e f}\left(\vec{r}^{\prime}, t\right) d t\right|^{2}+\kappa \int_{t}\left|s_{r e f}(\vec{r}, t)\right|^{2} d t \int_{t^{\prime}}\left|s_{r e f}\left(\vec{r}^{\prime}, t^{\prime}\right)\right|^{2} d t^{\prime} \\
& \approx \kappa N^{2} \delta\left(\vec{r}-\vec{r}^{\prime}\right)+\text { const. }
\end{aligned}
$$

where the field intensities for scalar, complex signals $f(\vec{r}, t)$ are defined as $|f(\vec{r}, t)|^{2}=f(\vec{r}, t)^{*} f(\vec{r}, t)$. In optical ghost imaging implementations, this condition motivates image formation with an approximately equivalent phaseless correlation of intensity fluctuations $G_{p l}(\vec{r})$ [19]:

$$
G_{p l}(\vec{r})=\int_{t}\left|s_{r e f}(\vec{r}, t)\right|^{2}\left|s_{R}(t)\right|^{2} d t-\kappa \int_{t}\left|s_{r e f}(\vec{r}, t)\right|^{2} d t \int_{t^{\prime}}\left|s_{R}\left(t^{\prime}\right)\right|^{2} d t^{\prime} .
$$

In the following, we will examine various requirements on phaseless imaging in an RCI setting. As in [1], we may discretize our reference and received waveforms into $M$ discrete increments $t_{i}, i=1, \ldots, M$ of length $1 / B W$, where $B W$ is the frequency bandwidth. For $Q=50$ pulses/positions, this results in $M=15,000$ total scattered field samples. Our region of interest can be discretized into $P$ pixels at positions $\vec{r}_{j}, j=1, \ldots, P$. Then we can write the forward model describing our measurements in Equation (3) as

$$
\mathbf{s}=\mathbf{H} \sigma
$$

where $\mathbf{s}$ is the $M \times 1$ vector of measurements, $s_{i}=s_{R}\left(t_{i}\right), \sigma$ is the $P \times 1$ vector describing the discretized scattering density, $\sigma_{j}=\sigma\left(\vec{r}_{j}\right)$, and the $M \times P$ matrix $\mathbf{H}$ is the matrix of reference fields with $H_{i j}=s_{r e f}\left(\vec{r}_{j}, t_{i}\right)$. Then the time-domain correlation of Equation (7) can be written as

$$
\sigma_{e s t}=\mathbf{H}^{\dagger} \mathbf{s} .
$$


where + denotes the conjugate transpose. Similarly, due to the equivalent expression of the covariance calculation in Equation (9) as

$$
G_{p l}(\vec{r})=\int_{t}\left[\left(\left|s_{r e f}(\vec{r}, t)\right|^{2}-\kappa \int_{t^{\prime}}\left|s_{r e f}\left(\vec{r}, t^{\prime}\right)\right|^{2} d t^{\prime}\right)\left(\left|s_{R}(t)\right|^{2}-\kappa \int_{t^{\prime \prime}}\left|s_{R}\left(t^{\prime \prime}\right)\right|^{2} d t^{\prime \prime}\right)\right] d t,
$$

we can write the phaseless correlation of Equation (9) as

$$
\hat{\sigma}_{e s t}=\hat{\mathbf{H}}^{\mathrm{T}} \hat{\mathbf{s}}
$$

where $\hat{\sigma}_{\text {est }}$ is the estimated intensity scattering response, and $\hat{\mathbf{H}}$ and $\hat{\mathbf{s}}$ consist of the reference and measured intensities, respectively:

$$
\begin{aligned}
& \hat{H}_{i j}=\left|H_{i j}\right|^{2}-\frac{1}{M} \sum_{i^{\prime}}^{M}\left|H_{i^{\prime} j}\right|^{2} \\
& \hat{s}_{i}=\left|s_{i}\right|^{2}-\frac{1}{M} \sum_{i^{\prime}}^{M}\left|s_{i^{\prime}}\right|^{2}
\end{aligned}
$$

Substitution of Equation (3) into Equation (7) reveals that the spatially-varying point spread function for the complex RCI system is given by the complex spatial autocorrelation of the reference fields, $\mathbf{H}^{\dagger} \mathbf{H}$. In a phaseless RCI system, the point spread function is given approximately (exactly, in the limit of an incoherent system [35,36]) by the intensity autocorrelation $\hat{\mathbf{H}}^{\mathrm{T}} \hat{\mathbf{H}}$. A significant result of this distinction is the loss of the ability to perform aperture synthesis through constellation motion in phaseless RCI. That is, phase contributions only occur in the phaseless system during the computation of the reference intensity pattern $\left|s_{r e f}(\vec{r}, t)\right|^{2}$ for each position. Since data from distinct trajectory positions is not coherently processed, the phaseless system point spread function is not sensitive to phase differences between the reference patterns corresponding to different positions in the trajectory. Instead, different trajectory positions contribute only by means of a much more slowly varying amplitude decay. This means that the resolving capabilities of the phaseless RCI system largely depend on the instantaneous array geometry, in contrast to the resolution in complex RCI which is determined by the total range of sampled positions achieved within the coherent integration time. This fact is illustrated in Figure 3.

Figure 3a illustrates a linear array oriented along the $y$ axis and moving along the $z$ direction. At each position along the constellation trajectory, all transmitting elements simultaneously emit a pulse, and the collective backscattered signal is measured by a single receiver. Figure $3 b$ shows the complex point spread function evaluated at the center of the region of interest, i.e., the image of a point reconstructed through Equation (7). The point is well-resolved in both directions due to the fact that the linear array combined with constellation motion has synthesized a two-dimensional array. In contrast, the result of phaseless reconstruction through Equation (9) is depicted in Figure 3c. Here, the point is resolved well along the direction of the linear array orientation, but is unresolved along the direction of array motion. These results correspond to the fact that while the phase diversity attained by the array along the $y$ direction yields significant interferometric variation in the intensities along that direction for a given constellation position, insufficient instantaneous phase variation is achieved in the reference signals along the $z$ direction, so that the phaseless system resolution in the $z$ direction is defined only by the amplitude decay induced by array motion. 
(a)

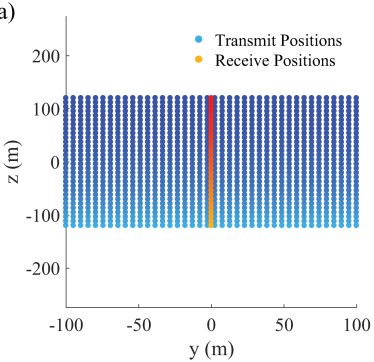

(d)

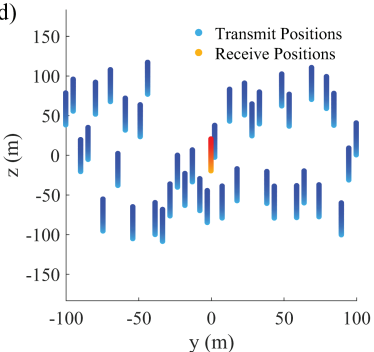

(b)

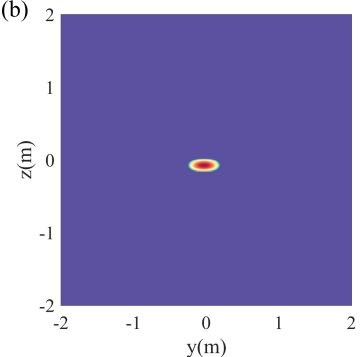

(e)

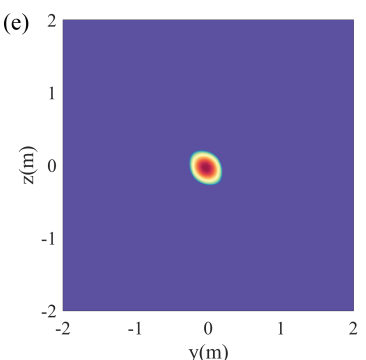

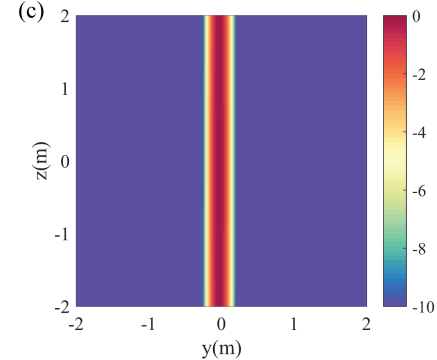

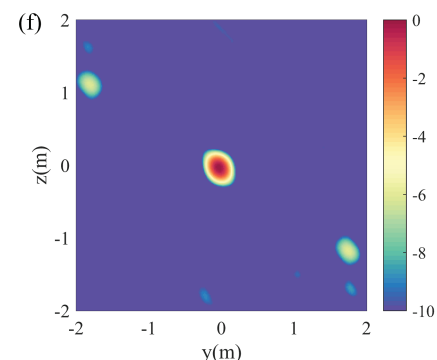

Figure 3. (a) Linear multiple-input single-output (MISO) array oriented along y direction and moving in $z$ direction. (b) Complex- correlation PSF for linear array. (c) Phaseless-correlation PSF for linear array. (d) Random MISO array moving along $z$ direction. (e) Complex-correlation PSF for random array. (f) Phaseless-correlation PSF for random array. Darker shades in $(\mathbf{a}, \mathbf{d})$ indicate increasing time as the constellation moves.

To remedy this disadvantage, we can modify the array so that its instantaneous geometry is two-dimensional at each position along the constellation trajectory. Figure $3 \mathrm{~d}$ illustrates this approach. In lieu of sparse array design (to be investigated in future works), the transmitting elements are randomly positioned over a two-dimensional synthetic aperture. In this way, the instantaneous reference signals generated from a single constellation position fluctuate significantly in amplitude and phase in both the $y$ and $z$ directions. The result is a well-resolved 2D image using both complex (Figure 3e) and phaseless (Figure 3f) reconstruction. We note that the point spread function resulting from the random array will be spatially-varying and dependent on the particular array realization. In the case shown, the resolution in the $z$ direction is worse than that of the linear array complex reconstruction, but this will not be true in general. From these results, we see that while the multistatic configuration in complex RCI offers an advantage mainly in terms of improved acquisition speed, such a geometry is fundamentally required to achieve high resolution in a phaseless RCI implementation.

In both the complex and phaseless implementations, accurate reconstruction relies on low spatial and temporal correlation of the interrogating reference fields [1,19]. The spatial correlation, given by $\mathbf{H}^{\dagger} \mathbf{H}$ (or $\hat{\mathbf{H}}^{\mathrm{T}} \hat{\mathbf{H}}$ ), and the temporal correlation, quantified by $\mathbf{H H}^{\dagger}$ (or $\hat{\mathbf{H}} \hat{\mathbf{H}}^{\mathrm{T}}$ ), can be jointly investigated through the singular value decomposition (SVD) of the $\mathbf{H}$ and $\hat{\mathbf{H}}$ matrices [37]. The SVD characterizes the number of distinct measurement modes achieved and the resulting conditioning of the measurement matrix. A flatter singular value spectrum generally indicates lower correlation between the spatial and temporal degrees of freedom in the reference signal. This semi-empirical design approach can be suitable for identifying sampling requirements when sparse, random arrays are considered [38]. Similar characterizations are used in compressive frameworks [39], which can potentially remedy inevitable undersampling (see Section 2.2.2 below). In Figure 2, we demonstrate the improved correlation properties of the reference fields as we increase the number of transmitters $N$ distributed throughout the array area. The magnitude of an example reference signal is plotted in Figure 2a for $N=10$, whereas Figure $2 \mathrm{~b}$ depicts a reference signal resulting from $N=40$ transmitters. A significant degree of spatial correlation is evident in the periodicity of the reference signal in Figure 2a. Such correlation is qualitatively seen to reduce in the signal of Figure $2 b$ generated using 40 transmitters. This fact is reflected by the flattening of the singular value spectra for $\mathbf{H}$ as $\mathrm{N}$ 
increases (Figure 2c). In practice, the singular value spectrum required to achieve successful imaging is determined by the noise floor and the space-bandwidth product of the system $[37,38]$. Using a single receiver, the geometry employed in these studies gives an expected resolution of $\delta_{y, z}=\frac{\lambda_{m} x}{D_{y, z}} \approx 0.31 \mathrm{~m}$ in the $y$ and $z$ directions, using the mean wavelength $\lambda_{m}$ and an approximate array size of $200 \mathrm{~m}$ in each direction. This yields a space-bandwidth product $S B P=\frac{A_{R O I}}{\delta_{y} \delta_{z}}$ of about 170 [40]. Satisfactory imaging requires this number of uncorrelated reference patterns. Since our aim is to generate random reference patterns through stochastic modulation instead of deterministic, orthogonal coding, our reference patterns will generally be correlated, leading to an imperfectly-conditioned $\mathbf{H}$ or $\hat{\mathbf{H}}$ matrix. This is reflected by the nonzero slope of the singular value spectrum, and generally incurs a requirement for more measurements to achieve successful imaging. Spatial correlation in the reference patterns is further illustrated by the sidelobes in the spatial autocorrelation plots in Figure 2d. These curves are obtained from a single row (here, corresponding to a center position in the region of interest) of the spatial autocorrelation matrix $\mathbf{H}^{\dagger} \mathbf{H}$, which serves as the point spread function in complex-valued RCI. Increasing $N$ results in generally reduced sidelobes in the spatial autocorrelation, indicating improved independence of the reference patterns. In the following, we employ $N=40$ transmitting elements.

As we have seen in the previous results, high fidelity image reconstruction depends on correlation of a received signal with spatially incoherent, computed reference fields resulting from the interference of a collection of stochastic signals generated by independent transmitting elements. Accurate computation of these reference fields thus demands precise phase synchronization between the collection of transmitters. This requirement holds for both the complex and phaseless RCI systems, and has been investigated for more general MIMO systems in other works [15,16,41,42]. Key distinctions nevertheless arise when considering the phase synchronization and stability requirements throughout the measurement process. In particular, phaseless RCI places no demands on transmit-receive phase synchronization. While a correspondence must still be made between the received measurements and the corresponding computed reference fields (i.e., time synchronization is required), discarding phase synchronization between transmitter and receiver offers a degree of hardware simplification. In addition, as discussed in $[23,24]$, any phase error accumulated by the signal between the region of interest and the receiver has no effect on the reconstruction. This result is demonstrated in Figure 4 for the case of two point scatterers. Here, random phase error was added to the scattered fields at each position in the region of interest before propagating to the single receiver. Figure $4 \mathrm{a}$ shows the image that results from reconstructing by the complex correlation of Equation (7). The phase-sensitive nature of the complex approach yields a corrupted image in which the two scatterers are completely indistinguishable. Phaseless correlation, on the other hand, accurately reconstructs the targets, as seen in Figure $4 \mathrm{~b}$.
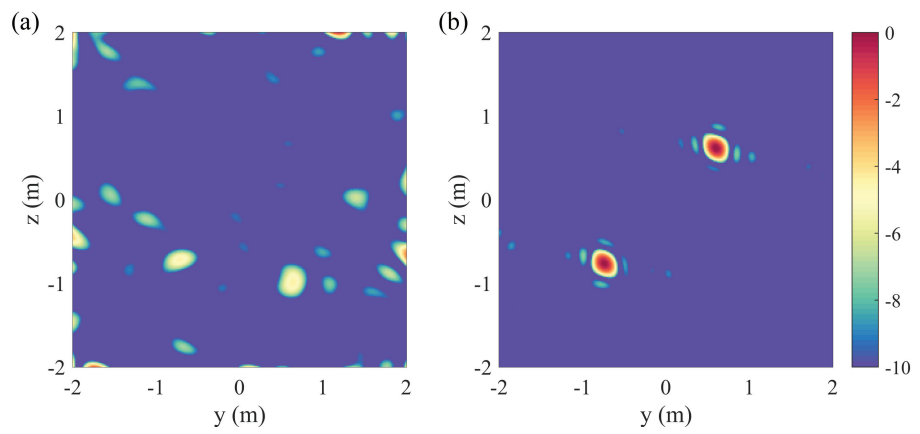

Figure 4. (a) Reconstruction by complex correlation with random phase error in scattered fields. (b) Reconstruction by phaseless correlation with random phase error in scattered fields.

Finally, a further advantage of phaseless reconstruction is a robustness to phase drift over the integration time. While relative phase between transmitting elements must continually be well characterized through synchronization methods, phase stability of each oscillator is only demanded 
over the span of a single pulse when employing phaseless reconstruction. This can offer a significant advantage when long integration times are required.

\subsubsection{Multiple Receivers}

The previous results treated the case of a single receiver that measured the combined backscatter resulting from all transmitting elements (MISO system). Building upon other RCI studies, we have examined the advantages of implementing incoherent processing techniques, derived from optical ghost imaging, in a microwave SAR setting. In this section, we investigate the extension of RCI to a MIMO system through a comparison of imaging results. We employ the same geometry as that shown in Figure 1, though allow multiple receivers to reside in the array. In the following MIMO simulations, we simply designate each array element to be a transceiver, so that $K=N=40$.

To accommodate MIMO acquisition in complex RCI, we modify the reconstruction operation to sum the temporal correlation over all received signals:

$$
G_{c}(\vec{r})=\sum_{k=1}^{K} \int_{t} s_{r e f, k}^{*}(\vec{r}, t) s_{R k}(t) d t
$$

The corresponding matrix operation can be achieved by concatenating the reference fields corresponding to each receiver along the row direction in the $\mathbf{H}$ matrix of Equation (11), and including the corresponding measurements in the s vector. Phaseless RCI can be approached similarly:

$$
G_{p l}(\vec{r})=\sum_{k=1}^{K}\left[\int_{t}\left|s_{r e f, k}(\vec{r}, t)\right|^{2}\left|s_{R k}(t)\right|^{2} d t-\kappa \int_{t}\left|s_{r e f, k}(\vec{r}, t)\right|^{2} d t \int_{t^{\prime}}\left|s_{R k}\left(t^{\prime}\right)\right|^{2} d t^{\prime}\right] .
$$

This expression can be simplified by observing the equivalent frequency-domain correlation, performed over the operating bandwidth and slow time interval:

$$
\begin{aligned}
G_{p l}(\vec{r})=\sum_{k=1}^{K}\left[\int_{t_{s}} \int_{f}\left|S_{r e f, k}\left(\vec{r}, f, t_{s}\right)\right|^{2}\left|S_{R k}\left(f, t_{s}\right)\right|^{2} d f d t_{s}\right. \\
\\
\left.\quad-\kappa^{\prime} \int_{t_{s}} \int_{f}\left|S_{r e f, k}\left(\vec{r}, f, t_{s}\right)\right|^{2} d f d t_{s} \int_{t_{s}^{\prime}} \int_{f^{\prime}}\left|S_{R k}\left(f^{\prime}, t_{s}\right)\right|^{2} d f^{\prime} d t_{s}^{\prime}\right]
\end{aligned}
$$

for $\kappa^{\prime}=\left(\int_{t_{s}} \int_{f} d f d t_{s}\right)^{-1}$, and noting

$$
\begin{aligned}
\left|S_{r e f, k}\left(\vec{r}, f, t_{s}\right)\right|^{2} & =\left|\sum_{n=1}^{N} S_{n}\left(f, t_{s}\right) \frac{\exp \left[-\frac{j 2 \pi f}{c}\left\{\left|\vec{r}-\vec{r}_{T n}\left(t_{s}\right)\right|+\left|\vec{r}_{R k}\left(t_{s}\right)-\vec{r}\right|\right\}\right]}{\left|\vec{r}-\vec{r}_{T n}\left(t_{s}\right)\right|\left|\vec{r}_{R k}\left(t_{s}\right)-\vec{r}\right|}\right|^{2} \\
& =\left|\sum_{n=1}^{N} S_{n}\left(f, t_{s}\right) \frac{\exp \left[-\frac{j 2 \pi f}{c}\left|\vec{r}-\vec{r}_{T n}\left(t_{s}\right)\right|\right]}{\left|\vec{r}-\vec{r}_{T n}\left(t_{s}\right)\right|\left|\vec{r}_{R k}\left(t_{s}\right)-\vec{r}\right|}\right|^{2}
\end{aligned}
$$

Finally, making the approximation that $\left|\vec{r}_{R k}\left(t_{s}\right)-\vec{r}\right|$ is nearly constant over the constellation, the phaseless RCI image can be calculated, neglecting constant amplitude terms, as

$$
\begin{aligned}
G_{p l}(\vec{r})= & \int_{t_{s}} \int_{f}\left[\left|\sum_{n=1}^{N} S_{n}\left(f, t_{s}\right) \frac{\exp \left[-\frac{j 2 \pi f}{c}\left|\vec{r}-\vec{r}_{T n}\left(t_{s}\right)\right|\right]}{\left|\vec{r}-\vec{r}_{T n}\left(t_{s}\right)\right|}\right|^{2}\right]\left[\sum_{k=1}^{K}\left|S_{R k}\left(f, t_{s}\right)\right|^{2}\right] d f d t_{s}- \\
& \kappa^{\prime} \int_{t_{s}} \int_{f}\left|\sum_{n=1}^{N} S_{n}\left(f, t_{s}\right) \frac{\exp \left[-\frac{j 2 \pi f}{c}\left|\vec{r}-\vec{r}_{T n}\left(t_{s}\right)\right|\right]}{\left|\vec{r}-\vec{r}_{T n}\left(t_{s}\right)\right|}\right|^{2} d f d t_{s} \int_{t_{s}^{\prime}} \int_{f^{\prime}} \sum_{k=1}^{K}\left|S_{R k}\left(f^{\prime}, t_{s}\right)\right|^{2} d f^{\prime} d t_{s}^{\prime},
\end{aligned}
$$

That is, we can simply sum the scattered intensities over the receiving aperture and use this summed intensity in the reconstruction. This correlation operation is equivalent to that employed in typical incoherent, optical ghost imaging, where spatial averaging of the scattered signal is 
performed by an optically large bucket detector [35]. In this way, we achieve a microwave equivalent to intensity-averaging bucket detection, with the result that we can realize incoherent SAR imaging in which the measured intensities are related linearly to the squared magnitude of the scattering density $|\sigma(\vec{r})|^{2}$. We see that the correlation is taken with respect to a new set of reference fields consisting of the intensities of the transmitted fields at the scene locations. Just as phaseless MISO RCI required no phase synchronization between the transmitters and receiver, the transmitters in phaseless MIMO RCI need not be phase synchronized with the collection of receivers. Moreover, no phase synchronization is required between receivers, as the signals are processed incoherently.

Figure 5 highlights the improved resolving capabilities inherent to MIMO signal acquisition. In complex RCI, this results from the broadening of the coherent imaging system transfer function through the convolution of the transmitting aperture's Fourier domain coverage with that of the receiving aperture [36,38,43]. Phaseless MIMO RCI, through incoherent processing as in Equation (19), achieves a similar resolution enhancement. Specifically, it has been shown [35] that in the limit of an infinitely large receiving aperture (approximately achieved as we increase $K$ ), the imaging process becomes incoherent, so that the point spread function is the squared magnitude of that of a system employing a single point receiver. In this case, the incoherent transfer function is given by the autocorrelation of the transmitting aperture's Fourier domain coverage, leading to a doubling of the transverse support in the spatial frequency domain $[36,44]$. Figure $5 \mathrm{a}, \mathrm{b}$ demonstrates complex and phaseless RCI reconstructions of two closely-spaced, sub-resolution points using signals gathered with a single receiver (MISO). Clearly, the distinct points are not resolvable under such a configuration. The case of MIMO acquisition with complex, coherent reconstruction is shown in Figure 5c, and with phaseless, incoherent reconstruction in Figure $5 \mathrm{~d}$. These images reveal the expected resolution improvement as the two points are now distinguishable. This transition is further emphasized in Figure 5e, where cross-sections along the $y$ direction are plotted for MISO and MIMO, complex and phaseless reconstruction.
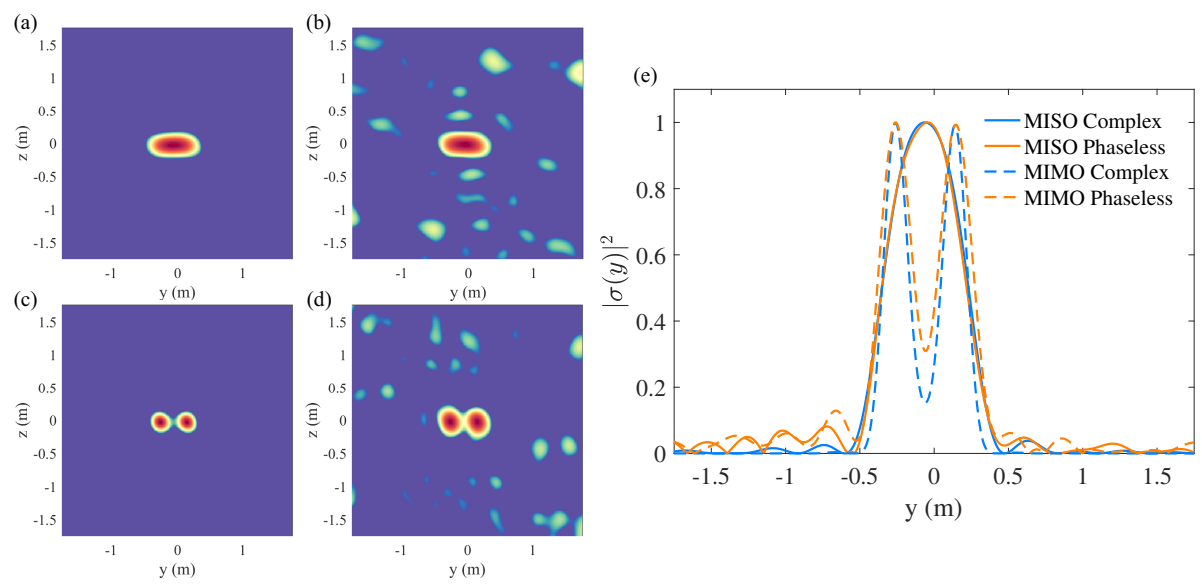

Figure 5. Image of two closely-spaced point targets reconstructed using (a) complex MISO RCI, (b) phaseless MISO RCI, (c) complex MIMO RCI, and (d) phaseless MIMO RCI. (e) $y$ cross-sections of two closely-spaced points reconstructed with complex and phaseless, MISO and MIMO RCI.

Figure 6 compares the imaging performance for the above cases of complex and phaseless, MISO and MIMO RCI. The simulated dataset consists of 50 pulses transmitted at equispaced positions as the random constellation moves $40 \mathrm{~m}$ in the $z$ direction. The scattered signals were received either by a single (MISO) receiver or by all (MIMO) transceivers. A noiseless scenario is considered here to compare the various reconstruction methods. Here, in lieu of the correlation operations described above, reconstruction of the extended airplane target is achieved by applying the pseudoinverse of the respective measurement matrices to the received datasets [45]. Figure $6 \mathrm{a}, \mathrm{b}$ depict the results for complex and phaseless MISO RCI, respectively. The qualitative target shape is reconstructed well by complex RCI, whereas the target is nearly indistinguishable in phaseless RCI with a single receiver. 
MIMO acquisition results shown in Figure $6 c$,d illustrate improved fidelity and resolution under complex reconstruction, and drastically improved image quality for phaseless reconstruction. Indeed, since the intensity averaging performed with phaseless MIMO acquisition essentially linearizes the problem as the system becomes incoherent, pseudoinversion applied to the measured intensities is further justified.
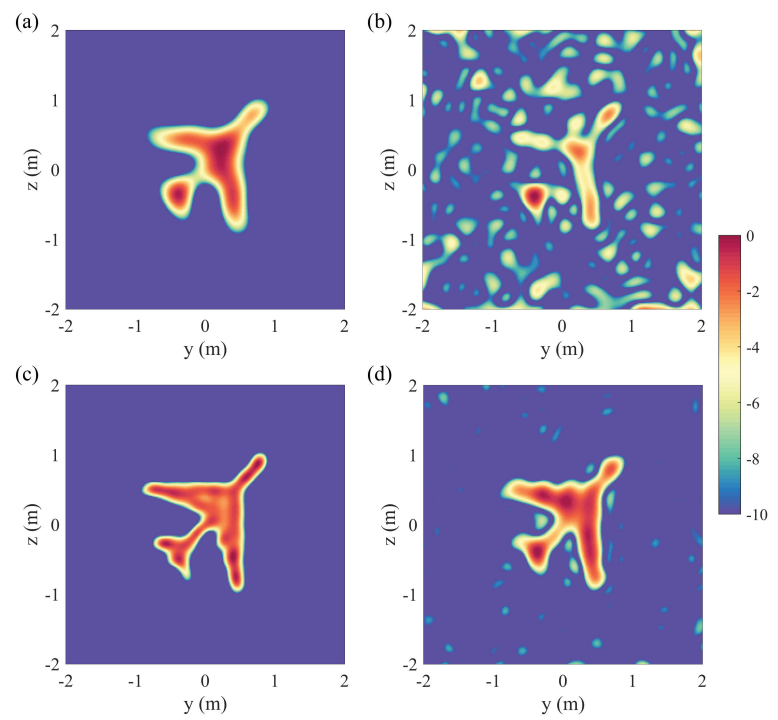

Figure 6. Example RCI images of an extended airplane target using a (a) complex MISO, (b) phaseless MISO, (c) complex MIMO, and (d) phaseless MIMO configuration.

The improved image fidelity observed in Figure $6 \mathrm{c}, \mathrm{d}$ follows in part simply from the $K$-fold increase in the number of measurements as the number of receivers increases from 1 to $K$. For the case of complex correlation, the computational cost thus increases by a factor of $K$. In contrast, utilizing the approximate phaseless, MIMO correlation given in Equation (19) incurs no additional computational cost beyond the extra step of averaging the received signals. As the size of the region of interest grows in practical scenarios, these savings can become increasingly advantageous.

In addition to increased computational requirements, larger regions of interest will necessitate more measurements and transmitter modules to achieve satisfactory image quality, as mentioned previously. Since this will inevitably be the case in practice, we briefly consider two known reconstruction approaches that can further alleviate data size and array density requirements. The first, called gradient ghost imaging (GGI), reduces the number of required measurements by directly reconstructing the edges or gradient of a target [46]. For a given set of measured signals, this improves the image SNR by reducing the effective size of the target [19,47]. Signal acquisition entails leveraging motion of the constellation to probe the region of interest with two sets of measurement patterns that are related simply by a transverse shift. A set of $M$ patterns paired with $M$ identical, but shifted, patterns thus results in a total of $2 M$ measurements. For each pair of shifted patterns, the difference in the measured intensities can be computed, and this set of difference measurements correlated with one of the set of $M$ patterns to obtain a gradient image. Figure $7 \mathrm{a}, \mathrm{b}$ compare this acquisition/reconstruction approach to that of phaseless RCI for a $4 \mathrm{~m}$ by $4 \mathrm{~m}$ scene. For these results, signals were taken as the random constellation moved $40 \mathrm{~m}$ along the $z$ direction. At each of 25 equispaced positions along the trajectory, a modulated pulse was transmitted and received by each element of the MIMO array. Then, at a distance of $13 \mathrm{~cm}$ from these positions, an identical pulse was transmitted and received, resulting in the interrogation of the scene with a shifted intensity pattern. Figure 7a depicts an image obtained through phaseless RCI processing using pseudoinversion of the phaseless dataset. In this case, distinct pulses were transmitted at each of the 50 total positions, for $2 M$ unique measurements. GGI processing of the target, shown in Figure $7 \mathrm{~b}$, achieves a gradient image with noticeably improved SNR. 
An additional approach that can diminish artifacts resulting from reduced datasets and sparse arrays is compressive reconstruction [39,48-51]. Figure 7c,d illustrate the results of applying TwIST [52] to the datasets described for Figure $7 \mathrm{a}, \mathrm{b}$. An $l_{1}$ regularizer was used to obtain these results, and the solution was assumed sparse in the pixel basis. Both the phaseless RCI and gradient images are more clearly distinguished using compressive reconstruction, with significant reduction in background noise. We note that since the gradient image will in general be more sparse in the pixel basis than its corresponding RCI result, GGI paired with compressive reconstruction may be a powerful combination for alleviating measurement and sampling requirements.
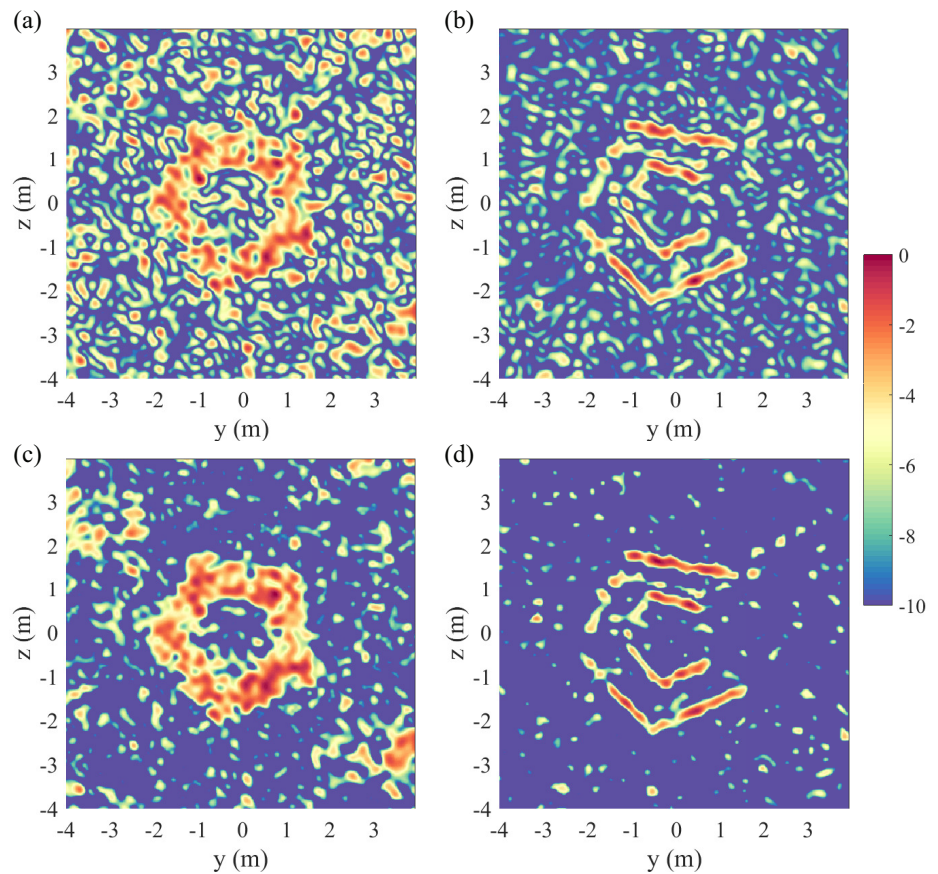

Figure 7. Example phaseless MIMO RCI images of a pentagon structure using (a) pseudoinversion, (b) GGI pseudoinversion, (c) TwIST, and (d) TwIST applied to GGI data.

\section{Discussion}

In this paper, we have extended the formulation of RCI to a fully MIMO configuration. Although we explored details of the algorithm in the context of airborne SAR, the results are readily extended to ground-based and spaceborne MIMO radar platforms. We also introduced phaseless microwave $\mathrm{RCI}$ as an incoherent processing alternative, with the potential for reduced sensitivity and synchronization requirements. We showed that despite the different approaches to processing the complex and phaseless datasets, both achieved similar performance improvements under MIMO acquisition in terms of image quality and resolution. Though coherent processing with complex data generally requires fewer measurements for satisfactory imaging, we have seen that phaseless RCI offers advantages through reduced synchronization demands between transmitting and receiving elements, robustness to phase error on the receive path, reduced computational demands, and compatibility with advanced reconstruction techniques such as gradient ghost imaging. Noiseless data was considered in all cases in order to isolate the effects of different modalities on the resulting reconstructions. While imaging performance will inevitably degrade with noise as in any other system, we point out two advantages inherent to the MIMO RCI scheme. The first is that the acquisition approach of simultaneous illumination by $N$ transmitters naturally yields a signal-to-noise ratio improvement, termed the Fellgett advantage [53], that is a well-known benefit of any multiplexing imaging system. The second is that the summing of the scattered intensities over $K$ receivers in the phaseless MIMO $\mathrm{RCI}$ implementation will result in signal averaging which yields an improved signal-to-noise ratio 
for each data point. These qualities can make MIMO RCI particularly robust to noise. Studies on the effect of noise and other implementation specifics such as misalignment on MIMO RCI SAR will be an interesting future direction of this work. As mentioned previously, our studies restricted the region of interest due to computational limitations. In practice, the antenna beamwidth and resulting spot size will determine the effective region of interest, which will necessitate longer measurement times and larger datasets. The trends reported here may dictate which strategies become most advantageous under such conditions, depending on dwell time and computational constraints. Finally, we have examined only two candidate methods for reconstructing from undersampled datasets. Improved performance can undoubtedly be achieved by employing a more appropriate sparse representation basis [39], though in practice this choice may depend on the targets of interest.

\section{Conclusions}

In this work we have generalized microwave RCI to accommodate MIMO acquisition scenarios with multiple transmitters and receivers. Whereas previous works have demonstrated coherent processing with perfectly synchronized array elements and complex data, we have introduced and examined incoherent RCI processing with phaseless data, for which synchronization requirements are relaxed. In addition, we have shown that incoherent RCI exhibits reduced sensitivity to phase error and lower computational complexity in a MIMO setting. We also showed that both coherent and incoherent RCI processing can benefit from MIMO operation in terms of image quality and resolution. Finally, we have demonstrated two related reconstruction methods that can be employed to further improve performance in undersampled conditions. These considerations can be important when evaluating trade-offs in a practical MIMO system.

Author Contributions: A.V.D. and M.F.I. conceived and designed the simulations; A.V.D. performed the simulations and analyzed the data; A.V.D., M.F.I. and D.R.S. wrote the paper.

Funding: This material is based upon work supported by the Air Force Office of Scientific Research under award number FA9550-12-1-0491 and FA9550-18-1-0187.

Conflicts of Interest: The authors declare no conflict of interest. The founding sponsors had no role in the design of the study; in the collection, analyses, or interpretation of data; in the writing of the manuscript, and in the decision to publish the results.

\section{Abbreviations}

The following abbreviations are used in this manuscript:

RCI Radar Coincidence Imaging

MIMO Multiple-Input Multiple-Output

MISO Multiple-Input Single-Output

SIMO Single-Input Multiple-Output

SAR Synthetic Aperture Radar

UAV Unmanned Aerial Vehicle

SVD Singular Value Decomposition

GGI Gradient Ghost Imaging

\section{References}

1. Li, D.; Li, X.; Qin, Y.; Cheng, Y.; Wang, H. Radar coincidence imaging: An instantaneous imaging technique with stochastic signals. IEEE Trans. Geosci. Remote Sens. 2014, 52, 2261-2277.

2. Zhu, S.; Zhang, A.; Xu, Z.; Dong, X. Radar coincidence imaging with random microwave source. IEEE Antennas Wirel. Propag. Lett. 2015, 14, 1239-1242. [CrossRef]

3. Li, D.; Li, X.; Cheng, Y.; Qin, Y.L.; Wang, H. Three dimensional radar coincidence imaging. Prog. Electromagn. Res. M 2013, 33, 223-238. [CrossRef]

4. Cheng, Y.; Zhou, X.; Xu, X.; Qin, Y.; Wang, H. Radar coincidence imaging with stochastic frequency modulated array. IEEE J. Sel. Top. Signal Process. 2017, 11, 414-427. [CrossRef] 
5. Zhu, S.; Dong, X.; Zhang, M.; Lu, R.; Li, J.; Chen, X.; Zhang, A. A Super-Resolution Computational Coincidence Imaging Method Based on SIMO Radar System. IEEE Geosci. Remote Sens. Lett. 2017, 14, 2265-2269. [CrossRef]

6. Soumekh, M. Synthetic Aperture Radar Signal Processing; Wiley: New York, NY, USA, 1999; Volume 7.

7. Bliss, D.; Forsythe, K. Multiple-input multiple-output (MIMO) radar and imaging: Degrees of freedom and resolution. In Proceedings of the IEEE Thirty-Seventh Asilomar Conference on Signals, Systems and Computers, Pacific Grove, CA, USA, 9-12 November 2003; Volume 1, pp. 54-59.

8. Haimovich, A.M.; Blum, R.S.; Cimini, L.J. MIMO radar with widely separated antennas. IEEE Signal Process. Mag. 2008, 25, 116-129. [CrossRef]

9. Krieger, G. MIMO-SAR: Opportunities and pitfalls. IEEE Trans. Geosci. Remote Sens. 2014, 52, $2628-2645$. [CrossRef]

10. Colin, J.M. Phased array radars in France: Present and future. In Proceedings of the IEEE International Symposium on Phased Array Systems and Technology, Boston, MA, USA, 15-18 October 1996; pp. 458-462.

11. Wang, W.Q. MIMO SAR OFDM chirp waveform diversity design with random matrix modulation. IEEE Trans. Geosci. Remote Sens. 2015, 53, 1615-1625. [CrossRef]

12. Kim, J.H.; Younis, M.; Moreira, A.; Wiesbeck, W. Spaceborne MIMO synthetic aperture radar for multimodal operation. IEEE Trans. Geosci. Remote Sens. 2015, 53, 2453-2466. [CrossRef]

13. Wang, W.Q. Large time-bandwidth product MIMO radar waveform design based on chirp rate diversity. IEEE Sens. J. 2015, 15, 1027-1034. [CrossRef]

14. Yang, Y.; Blum, R.S. Phase synchronization for coherent MIMO radar: Algorithms and their analysis. IEEE Trans. Signal Process. 2011, 59, 5538-5557. [CrossRef]

15. Godrich, H.; Haimovich, A.M.; Poor, H.V. An analysis of phase synchronization mismatch sensitivity for coherent MIMO radar systems. In Proceedings of the 3rd IEEE International Workshop on Computational Advances in Multi-Sensor Adaptive Processing (CAMSAP), Aruba, Dutch Antilles, The Netherlands, 13-16 December 2009; pp. 153-156.

16. Akçakaya, M.; Nehorai, A. MIMO radar detection and adaptive design under a phase synchronization mismatch. IEEE Trans. Signal Process. 2010, 58, 4994-5005. [CrossRef]

17. López-Dekker, P.; Mallorquí, J.J.; Serra-Morales, P.; Sanz-Marcos, J. Phase synchronization and Doppler centroid estimation in fixed receiver bistatic SAR systems. IEEE Trans. Geosci. Remote Sens. 2008, 46, 3459-3471. [CrossRef]

18. Gollub, J.; Yurduseven, O.; Trofatter, K.; Arnitz, D.; Imani, M.; Sleasman, T.; Boyarsky, M.; Rose, A.; Pedross-Engel, A.; Odabasi, H.; et al. Large metasurface aperture for millimeter wave computational imaging at the human-scale. Sci. Rep. 2017, 7, 42650. [CrossRef] [PubMed]

19. Gatti, A.; Bache, M.; Magatti, D.; Brambilla, E.; Ferri, F.; Lugiato, L. Coherent imaging with pseudo-thermal incoherent light. J. Mod. Opt. 2006, 53, 739-760. [CrossRef]

20. Shapiro, J.H. Computational ghost imaging. Phys. Rev. A 2008, 78, 061802. [CrossRef]

21. Bromberg, Y.; Katz, O.; Silberberg, Y. Ghost imaging with a single detector. Phys. Rev. A 2009, 79, 053840. [CrossRef]

22. Goodman, J.W. Statistical Optics; John Wiley \& Sons: Hoboken, NJ, USA, 2015.

23. Hardy, N.D.; Shapiro, J.H. Computational ghost imaging versus imaging laser radar for three-dimensional imaging. Phys. Rev. A 2013, 87, 023820. [CrossRef]

24. Hardy, N.D.; Shapiro, J.H. Reflective ghost imaging through turbulence. Phys. Rev. A 2011, 84, 063824. [CrossRef]

25. Erkmen, B.I. Computational ghost imaging for remote sensing. JOSA A 2012, 29, 782-789. [CrossRef] [PubMed]

26. Maci, S.; Minatti, G.; Casaletti, M.; Bosiljevac, M. Metasurfing: Addressing waves on impenetrable metasurfaces. IEEE Antennas Wirel. Propag. Lett. 2011, 10, 1499-1502. [CrossRef]

27. Gonzalez-Ovejero, D.; Chahat, N.; Sauleau, R.; Chattopadhyay, G.; Maci, S.; Ettorre, M. Additive Manufactured Metal-Only Modulated Metasurface Antennas. IEEE Trans. Antennas Propag. 2018, 66, 6106-6114. [CrossRef]

28. Sleasman, T.; F. Imani, M.; Gollub, J.N.; Smith, D.R. Dynamic metamaterial aperture for microwave imaging. Appl. Phys. Lett. 2015, 107, 204104. [CrossRef] 
29. Boyarsky, M.; Sleasman, T.; Pulido-Mancera, L.; Fromenteze, T.; Pedross-Engel, A.; Watts, C.M.; Imani, M.F.; Reynolds, M.S.; Smith, D.R. Synthetic aperture radar with dynamic metasurface antennas: A conceptual development. JOSA A 2017, 34, A22-A36. [CrossRef] [PubMed]

30. Watts, C.M.; Pedross-Engel, A.; Smith, D.R.; Reynolds, M.S. X-band SAR imaging with a liquid-crystal-based dynamic metasurface antenna. JOSA B 2017, 34, 300-306. [CrossRef]

31. Sleasman, T.; Boyarsky, M.; Pulido-Mancera, L.; Fromenteze, T.; Imani, M.F.; Reynolds, M.S.; Smith, D.R. Experimental Synthetic Aperture Radar with Dynamic Metasurfaces. IEEE Trans. Antennas Propag. 2017, 65, 6864-6877. [CrossRef]

32. Smith, D.R.; Yurduseven, O.; Mancera, L.P.; Bowen, P.; Kundtz, N.B. Analysis of a waveguide-fed metasurface antenna. Phys. Rev. Appl. 2017, 8, 054048. [CrossRef]

33. Skolnik, M.I. Introduction to Radar Systems; McGraw Hill Book Co.: New York, NY, USA, 1980.

34. Diebold, A.V.; Imani, M.F.; Sleasman, T.; Smith, D.R. Phaseless computational ghost imaging at microwave frequencies using a dynamic metasurface aperture. Appl. Opt. 2018, 57, 2142-2149. [CrossRef] [PubMed]

35. Cheng, J. Transfer functions in lensless ghost-imaging systems. Phys. Rev. A 2008, 78, 043823. [CrossRef]

36. Diebold, A.V.; Imani, M.F.; Sleasman, T.; Smith, D.R. Phaseless coherent and incoherent microwave ghost imaging with dynamic metasurface apertures. Optica 2018, 5, 1529-1541. [CrossRef]

37. Brady, D.J. Optical Imaging and Spectroscopy; John Wiley \& Sons: Hoboken, NJ, USA, 2009.

38. Marks, D.L.; Gollub, J.; Smith, D.R. Spatially resolving antenna arrays using frequency diversity. JOSA $A$ 2016, 33, 899-912. [CrossRef] [PubMed]

39. Lustig, M.; Donoho, D.; Pauly, J.M. Sparse MRI: The application of compressed sensing for rapid MR imaging. Magn. Reson. Med. Off. J. Int. Soc. Magn. Reson. Med. 2007, 58, 1182-1195. [CrossRef] [PubMed]

40. Lohmann, A.W.; Dorsch, R.G.; Mendlovic, D.; Zalevsky, Z.; Ferreira, C. Space-Bandwidth product of optical signals and systems. JOSA A 1996, 13, 470-473. [CrossRef]

41. He, Q.; Blum, R.S. Cramer-Rao bound for MIMO radar target localization with phase errors. IEEE Signal Process. Lett. 2010, 17, 83-86.

42. Krieger, G.; Moreira, A. Spaceborne bi-and multistatic SAR: Potential and challenges. IEE Proc.-Radar Sonar Navig. 2006, 153, 184-198. [CrossRef]

43. Ahmed, S.S. Electronic Microwave Imaging with Planar Multistatic Arrays; Logos Verlag Berlin GmbH: Berlin, Germany, 2014.

44. Goodman, J.W. Introduction to Fourier Optics; Roberts and Company Publishers: Englewood, CO, USA, 2005.

45. Zhang, C.; Guo, S.; Cao, J.; Guan, J.; Gao, F. Object reconstitution using pseudo-inverse for ghost imaging. Opt. Express 2014, 22, 30063-30073. [CrossRef] [PubMed]

46. Liu, X.F.; Yao, X.R.; Lan, R.M.; Wang, C.; Zhai, G.J. Edge detection based on gradient ghost imaging. Opt. Express 2015, 23, 33802-33811. [CrossRef] [PubMed]

47. Gureyev, T.; Paganin, D.; Kozlov, A.; Nesterets, Y.I.; Quiney, H. Complementary aspects of spatial resolution and signal-to-noise ratio in computational imaging. Phys. Rev. A 2018, 97, 053819. [CrossRef]

48. Katz, O.; Bromberg, Y.; Silberberg, Y. Compressive ghost imaging. Appl. Phys. Lett. 2009, 95, 131110. [CrossRef]

49. Eldar, Y.C.; Kutyniok, G. Compressed Sensing: Theory and Applications; Cambridge University Press: Cambridge, UK, 2012.

50. Potter, L.C.; Ertin, E.; Parker, J.T.; Cetin, M. Sparsity and compressed sensing in radar imaging. Proc. IEEE 2010, 98, 1006-1020. [CrossRef]

51. Cetin, M.; Stojanovic, I.; Onhon, O.; Varshney, K.; Samadi, S.; Karl, W.C.; Willsky, A.S. Sparsity-driven synthetic aperture radar imaging: Reconstruction, autofocusing, moving targets, and compressed sensing. IEEE Signal Process. Mag. 2014, 31, 27-40. [CrossRef]

52. Bioucas-Dias, J.M.; Figueiredo, M.A. A new TwIST: Two-step iterative shrinkage/thresholding algorithms for image restoration. IEEE Trans. Image Process. 2007, 16, 2992-3004. [CrossRef] [PubMed]

53. Gehm, M.E.; McCain, S.T.; Pitsianis, N.P.; Brady, D.J.; Potuluri, P.; Sullivan, M.E. Static two-dimensional aperture coding for multimodal, multiplex spectroscopy. Appl. Opt. 2006, 45, 2965-2974. [CrossRef] [PubMed]

(c) 2019 by the authors. Licensee MDPI, Basel, Switzerland. This article is an open access article distributed under the terms and conditions of the Creative Commons Attribution (CC BY) license (http:/ / creativecommons.org/licenses/by/4.0/). 